\title{
Effect of anti-retroviral therapy on oxidative stress in hospitalized HIV-infected adults with and without TB
}

\author{
Emmanuel Musisi ${ }^{1,8}$, Denis Kasozi Matovu ${ }^{8}$, Andrew Bukenya ${ }^{2}$, Sylvia Kaswabulii1, Josephine Zawedde ${ }^{1}$, \\ Alfred Andama ${ }^{1}$, Patrick Byanyima ${ }^{1}$, Ingvar Sanyu ${ }^{1}$, Abdul Sessolo ${ }^{1}$, Emmanuel Seremba ${ }^{3}$ J Lucian Davis ${ }^{6}$, \\ William Worodria ${ }^{1}$, Laurence Huang ${ }^{1,4,5}$, Nicholas D Walter ${ }^{1,7}$, Harriet Mayanja-Kizza ${ }^{3}$
}

1. Infectious Diseases Research Collaboration, Plot 2C Nakasero Hill Road PO Box 7475, Kampala Uganda.

2. College of Health Sciences, Department of Medical Biochemistry, Makerere University, PO Box 7072, Kampala, Uganda.

3. College of Health Sciences, Department of Medicine, Makerere University PO Box 7072, Kampala, Uganda.

4. HIV, Infectious Diseases, and Global Medicine Division, University of California, San Francisco, San Francisco, CA, USA.

5. Division of Pulmonary and Critical Care Medicine, University of California, San Francisco, San Francisco, CA, USA.

6. Yale University, New Haven, CT, USA.

7. Pulmonary Section, Denver Veterans Affairs Medical Center, Denver, USA.

8. College of Natural Science Department of Biochemistry and Sports Sciences, Makerere University, P. O. Box 7072, Kampala, Uganda.

\section{Emails:}

Emmanuel Musisi - emusisi@idrc-uganda.org, Sylvia Kaswabuli - skaswabuli@idrc-uganda.org, Josephine Zawedde - jzawedde@idrc-uganda.org, Alfred Andama - andama.alf@gmail.com, Patrick Byanyima - pbyanyima@idrc-uganda.org, Ingvar Sanyu - singvar@idrc-uganda. org, Abdul Sessolo - asessolo@idrc-uganda.org, J Lucian Davis - lucian.davis@yale.edu, William Worodria - wordria@yahoo.com, Laurence Huang - Laurence.Huang@ucsf.edu, Nicholas D Walter - Nicholas.Walter@ucdenver.com

Andrew Bukenya - bukerah@yahoo.com, Emmanuel Seremba - eseremba@yahoo.com, Harriet Mayanja-Kizza - hmk@chs.mak.ac.ug, Denis Kasozi Matovu-dkasozi@cns.mak.ac.ug

\section{Abstract}

Background: HIV infection and opportunistic infections cause oxidative stress (OS), which is associated with tissue damage. Anti-retroviral therapy (ART) is used to treat HIV and decrease the risk of opportunistic infections, but it is unclear whether ART reduces OS. Association of ART with OS was investigated.

Methods: We stratified a convenience sample of frozen serum or plasma from HIV-infected, ART-naïve ( $\mathrm{n}=21)$; HIV-infected, ART-treated ( $n=14)$; HIV and PTB co-infected, ART-naïve ( $n=21)$; HIV and PTB co-infected, ART-treated $(n=25)$ patients. Controls $(n=21)$ were HIV-negative adults without TB symptoms. Concentration of OS markers namely: transaminases (ALT and AST), gamma glutamyl transpeptidase (GGT), albumin, total protein, malondialdehyde (MDA), vitamin $\mathrm{C}$, and total anti-oxidant status (TAS) were determined.

Results: AST $(\mathrm{p}<0.001)$, GGT $(\mathrm{p}<0.001)$, total protein $(\mathrm{p}=0.001)$ and MDA $(\mathrm{p}<0.001)$ were higher in HIV patients compared to controls. Vitamin $C(P<0.0001)$ and albumin $(\mathrm{p}<0.01)$ were lower in HIV-patients relative to controls. ART was only associated with higher albumin $(\mathrm{p}=0.001)$, higher GGT $(\mathrm{p}=0.02)$ and lower vitamin $\mathrm{C}(\mathrm{p}=0.009)$. HIV and PTB co-infection was only significantly associated with higher GGT $(\mathrm{p}=0.01)$ and AST $(\mathrm{p}=0.03)$.

Conclusion: We identified severe OS among HIV-patients. ART was associated with both increased and reduced markers of OS hence suggesting that ART may not attenuate OS.

Keywords: Human immunodeficiency virus, Mycobacterium tuberculosis, Oxidative stress, anti-retroviral therapy, hospitalized patients.

DOI: https://dx.doi.org/10.4314/ahs.v18i3.7

Cite as: Musisi E, Matovu DK, Bukenya A, Kaswabuli S, Zawedde J, Andama A, Byanyima P, Sanyu I, Sessolo A, Seremba E, Davis JL, Worodria W, Huang L, Walter ND, Mayanja-Kizৃa H. Effect of anti-retroviral therapy on oxidative stress in Hospitalized HIV infected adults with and without TB. Afri Health Sci. 2018;18(3): 512-522. https://dx.doi.org/10.4314/abs.v18i3.7

\section{Corresponding author:}

Emmanuel Musisi, Infectious Diseases Research Collaboration, Plot 2C Nakasero Hill Road PO Box 7075, Kampala, Uganda

(Tel: +256 774080289/+256 752900396;

Email: emusisi@idrc-uganda.org

\section{Introduction}

Human immunodeficiency virus (HIV) and tuberculosis (TB) are the leading infectious causes of death worldwide $^{1}$. Globally, about 1.2 million people died from HIV in 2014. Of these, 400,000 were co-infected with $\mathrm{TB}^{2}$. The burden of infections is highest in sub-Saharan Africa where over $74 \%$ of the world's HIV and TB co-infections occur $^{3}$. In 2016, about 1.4 million Ugandans were reported to have HIV, and 28,000 people died of AIDS ${ }^{1}$.

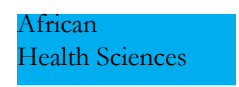

(C) 2018 Musisi et al. Licensee African Health Sciences. This is an Open Access article distributed under the terms of the Creative commons Attribution License (https://creativecommons.org/licenses/BY/4.0), which permits unrestricted use, distribution, and reproduction in any medium, provided the original work is properly cited. 
Oxidative stress (OS) is defined as a disturbance in the balance between the production of reactive oxygen species (i.e., free radicals) and anti-oxidant defenses. HIV infection is associated with increased production of free radicals leading to OS through a number of mechanisms ${ }^{4}$. HIV infection is also associated with suppression of the anti-oxidant defense system ${ }^{5,6} . M$. tuberculosis (Mtb) is the leading opportunistic infection among HIV patients and is also associated with OS. Mtb activates membrane-bound NADPH-oxidase, which leads to accumulation of reactive oxygen species ${ }^{7,8}$, leading to the respiratory burst and, subsequently, OS. OS predisposes to intracellular defects such as DNA damage leading to malignancies; accelerated apoptosis leading to metabolic disorders and premature ageing; neurological damage leading to dementia; increased HIV replication leading to immune dysfunction ${ }^{9-12}$, and poor treatment outcomes. OS also predicts mortality in HIV-infected patients ${ }^{13}$.

The use of ART has converted HIV into a chronic and manageable disease. It has reduced mortality and cases of opportunistic infections among HIV-infected patients. The role of OS in modulating HIV infection is well-established $^{11}$, but how ART modifies OS remains unclear.. While one study reported that ART was associated with decreased $\mathrm{OS}^{14}$, others found that it was associated with increased $\mathrm{OS}^{15-17}$. Uganda is among the 30 high TB burden countries with adult HIV prevalence rate of $6.5^{1}$, yet there is a paucity of data regarding oxidative stress among HIV and TB-co-infected patients.

Uncertainity regarding the role of ART in OS limits recommendations to intervene with anti-oxidant(s) supplements to avert OS in HIV-infected patients. Thus, better understanding of the role of ART on OS may lead to interventions to improve outcomes.

In this study, we aimed to: (1) compare OS markers in HIV-infected persons to those without HIV, (2) compare OS in HIV/TB co-infected individuals to those with HIV-mono infection and, (3) assess the association of ART with OS among hospitalized HIV and HIV/PTB co-infected patients in Uganda.

\section{Methods}

Study design and setting: We conducted a cross-sec- tional study among consenting HIV-infected adults $(\geq 18$ years) that were admitted to Mulago Hospital in Kampala, Uganda with possible pneumonia as part of the Mulago Inpatient Non-invasive Diagnosis-International HIV-associated Opportunistic Pneumonias (MIND-IHOP) study ${ }^{18-21}$ from 2013-2015.

Inclusion criteria for the sub-study were: being HIV positive with or without TB disease, HIV positive with or without being on ART, HIV negative with no symptoms of chronic disease(s) including TB, and availability of sufficient plasma and/or serum samples.

Exclusion criteria for the sub-study were: missing ART status related information, being on anti-tuberculosis therapy, age above 45 years, alcohol consumption $(>10$ bottles for more than 4-times/week), and cigarette smoking (>10 cigarettes/day).

We selected a convenience sample of specimens from four HIV patient groups: (1) HIV-infected, ART-naïve, (2) HIV-infected, ART-treated, (3) HIV and PTB co-infected, ART-naïve; and (4) HIV and PTB co-infected, ART-treated. ART naïve were newly diagnosed HIV patients who were not under formal HIV management until the time of recruitment into the MIND-IHOP study. HIV infection was confirmed at time of enrollment and PTB infection was diagnosed by direct fluorescent microscopy (auramine-O staining) or GeneXpert MTB/RIF (Cepheid, Sunnyvale CA) with confirmation by Lowenstein-Jensen culture method. HIV was screened for using Abbott rapid diagnostic test (Abbott Japan Co ltd. Tokyo, Japan), and confirmed using a STAT-PAC test (Chembio diagnostic system, INC New York, USA). CD4 counts were measured using a BD FACScalibur machine (BD Sciences, San Jose CA). We defined ART-treated patients as those who self-reported adherence to ART for $\geq 6$ months, while ART-naïve patients were HIV-infected patients who had never started ART. We additionally enrolled healthy controls testing negative for HIV, hepatitis $\mathrm{B}$, and $\mathrm{C}$ and without TB symptoms at a local blood bank. To minimize confounders, we excluded cigarette smokers, alcohol drinkers, and persons who were above 45 years of age or who had already initiated TB treatment.

Oxidative stress (OS) assays: Stored serum $\left(-20^{\circ} \mathrm{C}\right)$ and plasma $\left(-80^{\circ} \mathrm{C}\right)$ were used. Selection of markers was 
based on their clinical significance, power to reflect OS, and feasibility of their assays in our laboratory setting. OS is associated with elevated levels of aspartate transaminase (AST), alanine transaminase (ALT), gamma glutamyl transaminase (GGT), total protein, and malondialdehyde (MDA). OS is also associated with decreased levels of serum albumin, vitamin $\mathrm{C}$, and total anti-oxidant status (TAS).

We determined levels of AST (U/L), ALT (U/L), GGT $(\mathrm{U} / \mathrm{L})$, and albumin $(\mathrm{mg} / \mathrm{ml})$ using a clinical chemistry automated analyzer (COBASR MIRA INTEGRA 400 plus; Roche diagnostics, GMBH, Germany) ${ }^{22}$. This analyzer is located at the clinical chemistry laboratory of $\mathrm{Mu}-$ lago Hospital Complex, the National Referral Hospital in Kampala, Uganda.

Total protein levels were determined by Biuret method ${ }^{23}$. A mixture of plasma, distilled water and Biuret reagent was homogenized and then incubated for 30 minutes at ambient temperature. Standard solution $(2500 \mu \mathrm{g} / 250 \mathrm{ml}$ albumin) was prepared in a similar way. Absorbances were read at $580 \mathrm{~nm}$. Total protein concentration was obtained from a standard curve.

Plasma levels of MDA $(\mu \mathrm{M})$ were determined by a thiobarbituric acid (TBA) method ${ }^{14}$. To $200 \mu$ l of plasma was added $100 \mu \mathrm{l}$ of TBA $(15 \mathrm{~g}) / \mathrm{TBA}(0.67 \mathrm{~g}) / 0.25 \mathrm{MHCl}$ $(50 \mathrm{ml})$ mixture, followed by $200 \mu \mathrm{l} 1 \mathrm{M} \mathrm{HCl}$. This mixture was heated at $95^{\circ} \mathrm{C}$ for 1 hour, cooled on ice, and then centrifuged at $3000 \mathrm{rpm}$ for 2 minutes. The supernatant was mixed with $200 \mu \mathrm{l}$ of $3 \mathrm{M} \mathrm{NaOH}$ and absorbance of the mixture was read (in duplicates) at $540 \mathrm{~nm}$ within 10 minutes. Concentration of MDA $(\mu \mathrm{M})$ was calculated using a factor $\left(1.56 \times 10^{5} \mathrm{M}-1 \mathrm{~cm}\right)$.

Levels of vitamin C (mg/dl) were determined by the acid phosphotungstate (PTA) method ${ }^{24}$. Equal volumes of plasma and color reagent (PTA) were incubated in the dark at ambient temperature for 30 minutes and then centrifuged at $3000 \mathrm{rpm}$ for 15 minutes. Absorbance of the supernatant was read at $700 \mathrm{~nm}$ against a reagent blank. Vitamin $\mathrm{C}$ concentration was obtained from the standard curve constructed using standard ascorbic acid (99\% ascorbic acid from BDH, laboratory supplies, Poole England).
Concentrations of TAS were determined (in duplicates) by using a modified ABTS (RANDOX NX2332) meth$\mathrm{od}^{25}$. Briefly, $50 \mu \mathrm{l}$ of the sample was mixed with $250 \mu \mathrm{l}$ of chromogen (R2). The mixture was incubated at $37^{\circ} \mathrm{C}$ for 5 minutes before its absorbance (A1) was read at $600 \mathrm{~nm}$.

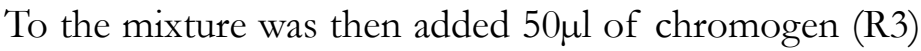
and incubated at $37^{\circ} \mathrm{C}$ for 3 minutes and its absorbance (A2) was read at $600 \mathrm{~nm}$. A reagent blank and standard were prepared in the same way but replacing the sample with double-distilled water and the standard, respectively. Difference in absorbance was calculated by subtracting A2 from A1 for all the three (blank, standard and samples). A factor was calculated by dividing the concentration of the standard by difference in absorbance between the blank and the standard. Concentrations of TAS were then calculated by multiplying the calculated factor with the difference in absorbance of the blank and that of the sample.

Statistical analysis: Statistical analysis was done using GraphPad Prism verion 4. We evaluated between-group differences using Mann-Whitney $\mathrm{U}$ test with an alpha level (p-value) of 0.05. We used one way ANOVA to compare differencesin age and gender between more than two groups. Exposures evaluated as risk factors for OS included HIV, PTB, age, gender and ART status.

\section{Results}

\section{Baseline characteristics}

This study included 102 patients: 81 who were HIV-infected and 21 who were HIV-negative healthy controls. Among the $81 \mathrm{HIV}$-infected, 21 were HIV-infected and ART-naïve, 14 were HIV-infected and ART-treated, 21 were HIV and PTB co-infected and ART-naïve, and 25 were HIV and PTB co-infected and ART-treated. The median age of all study participants was 35 (IQR 28-42) years and 53\% were women. Using One-way analysis of variance (ANOVA) test, we did not identify a statistically significant difference in gender $(p=0.18)$ and age $(\mathrm{p}=0.41)$ across study groups. Among the HIV-infected patients, the median CD4 T-cell count was 110 (IQR 34234) cells/ $\mu$ l. The median time on ART was 17.5 months among the HIV-mono-infected patients and 11.0 months among the HIV and PTB co-infected patients. Among HIV-infected patients, there was a non-significant trend towards higher CD4 $\mathrm{T}$ cells counts among those who were ART-treated relative to those who were ART-naive $(\mathrm{p}=0.24)$ (Table 1). 
Table 1. Baseline demographic and clinical characteristics of the participants

\begin{tabular}{|c|c|c|c|c|c|}
\hline $\begin{array}{l}\text { Participant } \\
\text { Characteristics }\end{array}$ & $\begin{array}{c}\text { HIV } \\
\text { (ART naive) }\end{array}$ & $\begin{array}{c}\text { HIV } \\
\text { (ART treated) }\end{array}$ & $\begin{array}{c}\text { HIV/PTB } \\
\text { (ART naive) }\end{array}$ & $\begin{array}{c}\text { HIV/PTB } \\
\text { (ART treated) }\end{array}$ & $\begin{array}{l}\text { Healthy } \\
\text { Controls }\end{array}$ \\
\hline Number (n) & 21 & 14 & 21 & 25 & 21 \\
\hline Female gender (\%) & $9(43 \%)$ & $8(57 \%)$ & $15(71 \%)$ & $11(46 \%)$ & $11(55 \%)$ \\
\hline Age (years) Median(IQR) & $31(22.5-39.0)$ & $39.5(27.5-48.0)$ & $32(27.5-37.5)$ & $38(32.50-45.5)$ & $30(27-33.0)$ \\
\hline 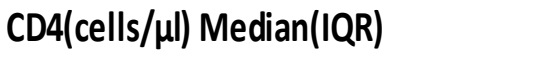 & $87(26-247.5)^{*}$ & $112(21.5-265.5)^{*}$ & $82(23-152.5)^{*}$ & $165(76.5-165.5)^{*}$ & $870(708-1037)$ \\
\hline ART duration (months) Median (IQR) & $N / A$ & $17.50(11-66)$ & N/A & $11(9.50-36)$ & N/A \\
\hline
\end{tabular}

Abbreviations definitions: ART=Antiretroviral therapy, HIV=Human immune deficiency syndrome, $\mathrm{TB}=$ Tuberculosis. Results are presented as percent (n) or Median (Interquartile range: IQR).* ${ }^{*}<0.001$; all in comparison with the reference group; N/A= not applicable

Oxidative stress markers in HIV-infected patients and HIV-negative healthy controls

Consistent with increased OS in HIV, the 81 HIV-infected patients combined had higher median AST (U/L) $[32.8$ vs 17.3; $\mathrm{p}<0.001]$, GGT (U/L) [64.0 vs 21.4; $\mathrm{p}<0.001$ ], total protein $(\mathrm{mg} / \mathrm{ml})[42.9$ vs $37.9 ; \mathrm{p}=0.012]$, and $\mathrm{MDA}$ $(\mu \mathrm{M})[1.73$ vs $1.37 ; \mathrm{p}<0.001]$ levels than the $21 \mathrm{HIV}$-negative controls. However, median ALT (U/L) level was lower in HIV-infected patients [9.0 vs 12.5; $\mathrm{p}=0.048$ ]. Also consistent with OS, HIV-infected patients in all groups combined had significantly lower median levels of albu$\min (\mathrm{g} / \mathrm{l})[29 \mathrm{vs} 44 ; \mathrm{p}<0.001]$ and vitamin C (mg/dl) [0.17 vs $0.51 ; \mathrm{p}<0.001]$. Although there was a trend towards lower median levels of TAS (mmol/l) in the HIV-infect- ed patients combined [1.6 vs $1.8 ; \mathrm{p}=0.99$ ], this was not statistically significant.

Association of ART with oxidative stress markers in all HIV-infected patients

As hypothesized, albumin $(\mathrm{g} / \mathrm{l})$ was higher among the HIV-infected ART-treated compared to the HIV-infected ART-naïve patients [33.3 vs 27.5; $\mathrm{p}=0.001]$. Median levels of AST, ALT, total protein, MDA, and TAS did not significantly $(p>0.05)$ differ between the ART-treated and the ART-naïve patients. The ART-treated had higher median levels of GGT (U/L) compared to the ART-naive patients [77.0 vs 53.0; $\mathrm{p}=0.023]$ and the median levels of vitamin C ( $\mathrm{mg} / \mathrm{dl})$ were significantly lower among the ART-treated compared to the ART-naive patients [0.25 vs $0.14 ; \mathrm{p}=0.01$ ] (Table 2 ). 
Table 2. Oxidative stress markers among ART-treated versus ART naïve patients

\begin{tabular}{|c|c|c|c|}
\hline Parameter & $\begin{array}{c}\text { HIV } \\
\text { (ART-treated)* }\end{array}$ & $\begin{array}{c}\text { HIV } \\
\text { (ART-naïve)* }\end{array}$ & $\begin{array}{c}\text { P-value } \\
\text { (statistical power) }\end{array}$ \\
\hline Vitamin C(mg/dl)Median(IQR) & $0.1(0.09-0.19)$ & $0.28(0.12-0.18)$ & $0.001(100)$ \\
\hline Albumin(g/I) Median(IQR) & $33.3(26.3-42.70)$ & 27.50(19.70-31.30) & 0.001 (100) \\
\hline TP(mg/ml) Median(IQR) & $43.8(39.5-45.40)$ & $42.90(40-45.30)$ & $0.66(68)$ \\
\hline TAS(mmol/I) Median(IQR) & $1.3(1.00-1.89)$ & $1.58(1.03-2.22)$ & $0.43(99.8)$ \\
\hline MDA( $\mu M)$ Median(IQR) & $1.7(1.46-1.96)$ & $1.73(1.56-1.86)$ & $0.88(83.4)$ \\
\hline ALT(IU) Median(IQR) & $9.9(5.9-17.5)$ & $8.65(5.75-12.70)$ & $0.32(100)$ \\
\hline AST(IU) Median(IQR) & $36.7(21.5-57.00)$ & $29.70(21.50-55.35)$ & $0.70(37.5)$ \\
\hline GGT(IU) Median(IQR) & $77.0(48.00-161)$ & $53(30-80.50)$ & $0.02(100)$ \\
\hline
\end{tabular}

Association of HIV mono-infection and HIV/PTB co-infection with oxidative stress markers

Median levels of AST (U/L) [43.5 vs 28.2; $\mathrm{p}=0.03]$ and GGT (U/L) [75 vs 42 ; $\mathrm{p}=0.01]$ were significantly higher among the HIV and PTB co-infected when compared with the HIV mono-infected patients. However, median levels of ALT, total protein, MDA, albumin, vitamin C, and TAS did not differ significantly ( $p>0.05$ ) between the HIV and PTB co-infected and, the HIV-mono-infected patients (Table 3).

Table 3. Oxidative stress markers in HIV mono-infected and HIV/TB co-infected patients

\begin{tabular}{|c|c|c|c|}
\hline Parameter & HIV mono infected* & HIV/PTB co-infected* & P-value \\
\hline Vitamin C(mg/dl) Median(IQR) & $0.16(0.11-0.35)$ & $0.19(0.09-0.41)$ & 0.76 \\
\hline Albumin (g/l) Median(IQR) & $28.2(19.0-34.45)$ & $30.1(25.00-40.85)$ & 0.14 \\
\hline TP (mg/ml) Median(IQR) & $42.9(39.45-46.90)$ & $43.2(37.40-45.30)$ & 0.78 \\
\hline TAS (mmol/I) Median(IQR) & $1.3(0.83-1.93)$ & $1.7(1.12-2.37)$ & 0.16 \\
\hline $\operatorname{MDA}(\mu \mathrm{M})$ Median(IQR) & $1.8(1.55-1.94)$ & $1.7(1.54-1.87)$ & 0.61 \\
\hline ALT(IU) Median(IQR) & $9.0(6.2-15.4)$ & $9.1(5.45-13.90)$ & 0.58 \\
\hline AST(IU) Median(IQR) & $28.2(20.65-42.20)$ & $43.5(23.65-66.85)$ & 0.03 \\
\hline GGT(IU) Median(IQR) & $42.0(22.5-66.00)$ & $75.0(45-147)$ & 0.01 \\
\hline
\end{tabular}


Association of ART with oxidative stress markers in HIV/PTB co-infected patients

Median vitamin $\mathrm{C}(\mathrm{mg} / \mathrm{dl})$ level was lower among the ART-treated HIV and PTB co-infected relative to the ART-naïve HIV and PTB co-infected patients [0.14 vs. $0.28 ; \mathrm{p}=0.029]$. However, median albumin $(\mathrm{g} / \mathrm{l})$ level was significantly higher [39.5 vs 25.5; $\mathrm{p}<0.001$ ] among the ART-treated HIV and PTB co-infected patients relative to the ART-naïve HIV and PTB co-infected patients. Median levels of AST, ALT, GGT, total protein, MDA, and TAS did not differ significantly $(\mathrm{p}>0.05)$ between the ART-treated HIV and PTB co-infected, and the ARTnaïve HIV and the PTB co-infected patients. (Table 4).

Table 4. Association of ART and OS markers in the HIV/PTB co-infected patients

\begin{tabular}{|c|c|c|c|}
\hline Parameter & HIV/PTB (ART treated) ${ }^{\beta}$ & HIV/PTB (ART naïve) ${ }^{\beta}$ & P-value \\
\hline Vitamin C (mg/dl) Median(IQR) & $0.14(0.10-0.17)$ & $0.28(0.12-0.41)$ & 0.03 \\
\hline Albumin (g/l) Median(IQR) & $39.5(27.9-48.90)$ & $25.5(21.80-31.30)$ & 0.001 \\
\hline TP (mg/ml) Median(IQR) & $44.0(37.65-45.35)$ & $41.8(37.10-44.80)$ & 0.39 \\
\hline TAS (mmol/I) Median(IQR) & $1.2(1.06-1.87)$ & $1.7(1.12-2.37)$ & 0.06 \\
\hline $\operatorname{MDA}(\mu \mathrm{M})$ Median(IQR) & $1.3(1.51-1.85)$ & $1.7(1.59-1.91)$ & 0.65 \\
\hline ALT(IU) Median(IQR) & $9.9(5.9-17.05)$ & $7.0(4.7-12.70)$ & 0.24 \\
\hline AST(IU) Median(IQR) & $39.2(24.45-57.30)$ & $54.2(22.70-72.20)$ & 0.29 \\
\hline GGT(IU) Median(IQR) & $77.5(45.0-180.0)$ & $70.0(46.50-172)$ & 0.95 \\
\hline
\end{tabular}

\section{Discussion}

In this study of hospitalized HIV and HIV/PTB co-infected patients in Uganda, we found that HIV-infected patients were under severe OS with both increased levels of OS markers and decreased levels of anti-oxidants. Overall, neither ART nor PTB were associated with a significant difference in levels of OS markers. This suggests that anti-oxidant supplementation might be beneficial irrespective of ART use.

As a group, HIV-infected patients had lower levels of plasma vitamin $\mathrm{C}$ and albumin and higher MDA relative to healthy controls, consistent with higher oxidative stress. Vitamin $\mathrm{C}$ scavenges free radicals and may be depleted due to increased free radical production in HIV/ PTB $^{26-28}$. HIV proteins such as negative regulatory factor (Nef), Viral protein $r(\mathrm{Vpr})$ and trans-activator of transcription (Tat) act as direct modulators of OS through up-regulating free radicals production and invasion of the antioxidant defense system ${ }^{29}$. MTB increases levels of free radicals through activation of the membrane bound NADPH oxidase and myeloperoxidase enzymes leading to respiratory burst. Additionally, the low levels of plasma vitamin $\mathrm{C}$ among the HIV and HIV/PTB co-infected patients may be explained by low dietary intake or malabsorption due to oxidative damage on the gastrointestinal tract ${ }^{30}$. Our findings are consistent with previous reports of low plasma vitamin $C$ and serum albumin levels in ambulatory HIV and PTB-infected individuals ${ }^{31-36}$. Importantly, low levels of vitamin $\mathrm{C}$ is a risk factor to certain cancers and scurvy ${ }^{37}$, while low serum albumin levels independently predict in-hospital death ${ }^{38,39}$. MDA is a product of lipid peroxidation that is caused by the free radicals attack on the polyunsaturated fatty acids in the cell membranes ${ }^{14}$. Higher levels of MDA in HIV and HIV/ PTB co-infected patients may hence indicate increased production of ROS molecules. MDA is transported by 
lipoproteins to distant tissues by circulation. There, MDA induces secondary production of ROS and subsequent uncontrolled lipid peroxidation. Over production of MDA through uncontrolled lipid peroxidation may cause systemic cell damage through altering the cell membrane fluidity. This may further compromise the immune system and lead to poor health of the HIV and HIV/PTB co-infected patients ${ }^{40-43}$.

Different populations on ART may have different OS levels due to differences in their lifestyle, environment pollutants and genetic variability. Conceptually, ART could reduce OS by reducing viral activity. However, ART drugs and/or their metabolic products may also augment OS by blocking the anti-oxidant-defense system ${ }^{44}$, or by activating the pathways that produce free radicals. Bechan Sharma proposed that ART or its metabolites could block three key anti-oxidant enzymes including superoxide dismutase, glutathione peroxidase, and glutathione reductase. Subsequently, this would lead to accumulation of ROS molecules such as superoxide anions, hydrogen peroxides $^{44}$.

Several previous studies have had conflicting findings regarding the association of ART with OS. Among ambulatory HIV patients in Nigeria, ART reduced OS $^{14}$. However, other studies showed that ART augments OS ${ }^{45-}$ 51. Enhanced OS during ART treatment may be due to altered oxidative phosphorylation and increased production of oxidized metabolites. Interestingly, the magnitude of ART induced OS seems to be differential and dependent on a number of factors including ART duration and adherence, and the composition of the therapeutic regimen $^{52-54}$. In the current study, we did not identify a significant association between ART and most of the OS markers, but we also did not have sufficient power to examine the impact of specific ART medications or ART duration on OS. This observation suggests that all HIV-infected patients, regardless of ART use, could potentially benefit from extrinsic anti-oxidant supplementation.

Hepatic markers reflect OS and end organ dysfunction although they are not yet regarded as cardinal markers of OS. GGT, total protein and liver transaminases (ALT and AST) have been suggested as inexpensive and easily as- sayed markers of OS in humans ${ }^{55}$ and in animal ${ }^{56}$ models. In this study, we observed higher levels of GGT, AST and total protein among HIV and HIV/PTB co-infected patients, which is consistent with increased OS regardless of the ART status. Being on ART was associated with significantly higher GGT among the HIV mono-infected but this was not the case among the PTB co-infected patients. The reason for this disparity was unclear to us. However, it could partly be attributed to ART duration. ART duration among the HIV-mono-infected was 6 months higher than that among the PTB co-infected patients. OS due to HIV and PTB infections or to chronic ART use may independently be associated with inflammatory reactions which may cause increased production of proteins mainly the globulins ${ }^{49}$, and oxidative liver tissue damage. This could explain the higher levels of GGT, AST and total protein that we observed in the HIV and $\mathrm{HIV} / \mathrm{PTB}$ co-infected patients.

This study has several limitations. Since this was a cross sectional study, we could not confirm OS causality. Our sample size of HIV mono-infected patients was small, potentially limiting our power to identify small effects. ART adherence was based on the patients' self-report, and may tend to over-estimate exposure. We were unable to assess the impact of variation in bacillary load and severity of TB disease. Some of the cardinal makers of OS such as glutathione, superoxide dismutase, and catalase, were not assessed. Nevertheless, our study provides important new insights into OS in HIV and HIV/TB co-infected patients in sub-Saharan Africa.

\section{Conclusion}

This study has demonstrated severe OS among hospitalized HIV-patients compared with those without HIV. We have shown that markers of OS do not significantly differ between HIV/PTB co-infected persons compared with those who were HIV mono-infected. We have also demonstrated that markers of OS do not significantly differ between ART treated persons and those who are ART naïve.

We hence recommend studies to explore the potential benefit of intervention with cost effective anti-oxidants supplementation in HIV patients. 
List of abbreviations: ABTS: 2, 2'-azino-bis-(3-methyl) benzothiozoline-6-sulfonate; ALT: Alanine amine transferase; AST: Aspartate amine transferase; DNA: Deoxyribonucleic acid; GGT: Gamma glutamyltranspeptidase; HCL: Hydrochloric acid; HIV: Human immunodeficiency virus; MDA: Malondialdehyde; NADPH: Reduced Nicotinamide adenosine diphosphate; OS: Oxidative stress; PTA: Phosphotungstate; TAS: Total anti-oxidant status; TBA: Thiobarbituric acid; TB: Tuberculosis; TP: Total protein.

\section{Declarations}

Ethics approval and consent to participate: Study protocol was approved by the Mulago Hospital Research Ethics Committee, the Makerere University School ofMedicine, Research Ethics Committee, the Uganda National Council for Science andTechnology, and the Committee on Human Research at the University of California San Francisco. All participants signed written informed consent.

\section{Consent for publication}

NA.

\section{Authors' contribution}

EM planned and coordinated the study, analyzed the data and prepared the manuscript. PB, SK, and AA oversaw sample collection. AS, IS, JZ identified and enrolled all patients; and collected all the clinical data. DKM, AB, ES, WW, LH, NDW and HMK oversaw the conduct of the study, data analysis and assisted with preparation of the manuscript. All authors critically reviewed the manuscript and approve of the submitted version.

\section{Acknowledgements}

The authors wish to acknowledge the patients who participated in this study; the staff and administration of Mulago Hospital; our colleagues Mr.Wamutu Samuel (Senior lecturer, Department of Biochemistry, Makarere University), the clinical, research, and administrative staff of the MU-UCSF Research Collaboration, especially Emily Chang, Catherine Nabakiibi and Yusuf Magezi; and the administrators of the MakCHS-UC Berkeley-Yale Pulmonary Complications of AIDS Research Training (PART) program.

African Health Sciences Vol 18 Issue 3, September, 2018

\section{Source of funding}

The MIND-IHOP study was funded by the IHOP grant (NIH R01 HL090335), Lung MicroCHIP grant (NIH U01 HL098964) and K24 grant (NIH K24 HL087713). These sources provided the funding to support subject enrollment and specimen collection. Dr. Nicholas D Walter provided financial support to buy the total anti-oxidant status kit. COHRE mentored research scholarship, Joint Clinical Research Collaboration, Kampala, Uganda and the HED-CIMTRADZ Project, COVAB-Makerere University provided financial support to buy the laboratory reagents. Emmanuel Musisi was supported by a scholarship from the Pulmonary Complications of AIDS Research Training Program (NIH D43TWOO9607). Funders had no influence in study design, data analysis and interpretation; and in manuscript write-up.

\section{Conflict of interest}

No conflict of interest declared.

\section{References}

1. World Health Organization WHO. Global tuberculosis report 2015. WHO/HTM/TB/2015.22. Geneva, WHO Press; 2015.

2. World Health Organization WHO. March 2014 supplement to the 2013 consolidated guidelines on the use of antiretroviral drugs for treating and preventing HIV infection: recommendations for a public health approach. 2014.

3. World Health Organization WHO. Global status report on alcohol and health. World Health Organization; 2014. 4. Jones DP. Radical-free biology of oxidative stress. American Journal of Physiology-Cell Physiology. 2008;295(4):C849-C868.

5. Cribbs SK, Guidot DM, Martin GS, Lennox J, Brown LA. Anti-retroviral therapy is associated with decreased alveolar glutathione levels even in healthy HIV-infected individuals. PloS one. 2014;9(2)

6. Nkechi OF, Chinedu OC, Ositadinma IM, Ebere AJ, Michael E, Priscilla EI. Impact of HIV and mycobacterium tuberculosis co-infections on anti-oxidant status in Nigeria. Pakistan Journal of Nutrition. 2013;12(5):496.

7. Shepherd VL. The role of the respiratory burst of phagocytes in host defense. Paper presented at: Seminars in respiratory infections 1986.

8. Slauch JM. How does the oxidative burst of macro- 
phages kill bacteria? Still an open question. Molecular microbiology. 2011;80(3):580-583.

9. Dobmeyer TS, Findhammer S, Dobmeyer JM, et al. Ex vivo induction of apoptosis in lymphocytes is mediated by oxidative stress: role for lymphocyte loss in HIV infection. Free Radical Biology and Medicine. 1997;22(5):775-785. 10. Kasapoglu B, Turkay C, Bayram Y, Koca C. Role of GGT in diagnosis of metabolic syndrome: A clinic-based cross-sectional survey. 2010.

11. Salmen S, Berrueta L. Immune modulators of HIV infection: the role of reactive oxygen species. Journal of Clinical \& Cellular Immunology. 2012;2012.

12. Valcour V, Shiramizu B. HIV-associated dementia, mitochondrial dysfunction, and oxidative stress. Mitochondrion. 2004;4(2):119-129.

13. Masiá M, Padilla S, Fernández M, et al. Oxidative Stress Predicts All-Cause Mortality in HIV-Infected Patients. PloS one. 2016;11(4)

14. Awodele O, Olayemi SO, Nwite JA, Adeyemo TA. "Investigation of the Levels of Oxidative Stress Parameters in HIV and HIV-TB Co-Infected Patients, "Journal of Infection in Developing Countries. 2012; 6 (1): 79- 85.”

15. Kwara A, Flanigan T, Carter E. Highly active antiretroviral therapy (HAART) in adults with tuberculosis: current status. The International Journal of Tuberculosis and Lung Disease. 2005;9(3):248-257.

16. Mandas A, Iorio EL, Congiu MG, et al. Oxidative imbalance in HIV-1 infected patients treated with antiretroviral therapy. BioMed Research International. 2009.

17. Martin JA, Sastre J, de la Asunción JG, Pallardó FV, Viña J. Hepatic $\gamma$-cystathionase deficiency in patients with AIDS. JAMA. 2001;285(11):1444-1445.

18. CattamanchiA, Davis JL, Worodria W, et al. Sensitivity and specificity of fluorescence microscopy for diagnosing pulmonary tuberculosis in a high HIV prevalence setting. The International Journal of Tuberculosis and Lung Disease. 2009;13(9):1130-1136.

19. Davis JL, Worodria W, Kisembo H, et al. Clinical and radiographic factors do not accurately diagnose smear-negative tuberculosis in HIV-infected inpatients in Uganda: a cross-sectional study. PLoS One. 2010;5(3)

20. Kyeyune R, den Boon S, Cattamanchi A, et al. Causes of early mortality in HIV-infected TB suspects in an East African referral hospital. Journal of acquired immune deficiency syndromes, (1999). 2010;55(4):446.

21. Yoo SD, Cattamanchi A, Den Boon S, et al. Clinical significance of normal chest radiographs among HIV-seropositive patients with suspected tuberculosis in Uganda. Respirology. 2011;16(5):836-841.

22.http://www.cobas.com/home/product/clinical-and-immunochemistry-testing/cobas-integra-400-plus.html.

23. Sapan CV, Lundblad RL, Price NC. Colorimetric protein assay techniques. Biotechnology and applied Biochemistry. 1999;29(2):99-108.

24. Kyaw A. A simple colorimetric method for ascorbic acid determination in blood plasma. Clinica chimica acta. 1978;86(2):153-157.

25. Gupta R, Sharma M, Lakshmy R, Prabhakaran D, Reddy KS. Improved method of total antioxidant assay. Indian Journal of Biochemistry \& Biophysics. 2009;46(1):126.

26. Allard JP, Aghdassi E, Chau J, et al. Effects of vitamin $\mathrm{E}$ and $\mathrm{C}$ supplementation on oxidative stress and viral load in HIV-infected subjects. Aids. 1998;12(13):16531659.

27. Bilbis LS, Idowu DB, Saidu Y, Lawal M, Njoku CH. Serum levels of anti-oxidant vitamins and mineral elements of human immunodeficiency virus positive subjects in Sokoto, Nigeria. Annals of African Medicine. 2010;9(4).

28. Mohod K, Dhok A, Kumar S. Status of oxidants and antioxidants in pulmonary tuberculosis with varying bacillary load. Journal of Experimental Sciences. 2011;2(6).

29. Fausther-Bovendo, H, Vieillard, et al. HIV gp41 engages $\mathrm{gC} 1 \mathrm{qR}$ on $\mathrm{CD}^{+}{ }^{+} \mathrm{T}$ cells to induce the expression of an NK ligand through the PIP3/H2O2 pathway. PLoS pathogens. 2010;6

30. Mukojima K, Mishima S, Oda J, et al. Protective Effects of Free Radical Scavenger Edaravone Against Xanthine Oxidase-Mediated Permeability Increases in $\mathrm{Hu}-$ man Intestinal Epithelial Cell Monolayer. Journal of Burn Care \& Research. 2009;30(2):335-340.

31. Matos E, Lemos ACM. Association between serum albumin levels and in-hospital deaths due to tuberculosis. The International Journal of Tuberculosis and Lung Disease. 2006;10(12):1360-1366.

32. Ngondi JL, Oben J, Forkah DM, Etame LH, Mbanya D. The effect of different combination therapies on oxidative stress markers in HIV infected patients in Cameroon. AIDS Research and Therapy. 2006;3(1):19.

33. Serpa J, Haque D, Valayam J, Breaux K, Rodriguez-Barradas, Maria C. Effect of combination antiretroviral treatment on total protein and calculated globulin 
levels among HIV-infected patients. International Journal of Infectious Diseases. 2010;14

34. Mohod K, Kumar S. Oxidants and Anti-oxidants Levels in Pulmonary Tuberculosis Patients on Anti-tubercular Treatment. Biomedical Research. 2012;23(3):385-389.

35. Mokondjimobe E, Longo-Mbenza B, Akiana J, et al. Biomarkers of oxidative stress and personalized treatment of pulmonary tuberculosis: emerging role of gamma-glutamyltransferase. Advances in pharmacological sciences. 2012.

36. Hansen SN, Tveden-Nyborg P, Lykkesfeldt J. Does vitamin $\mathrm{C}$ deficiency affect cognitive development and function? Nutrients. 2014;6(9):3818-3846.

37. Shirai T, Sato A, Chida K, et al. [A study of causes of death among patients with active pulmonary tuberculosis from the standpoint of host factors]. Kekkaku:[Tuberculosis]. 1990;65(6):397-405.

38. Yamanaka, K, Sakai S, Nomura F, Akashi T, Usui T. [A nutritional investigation of homeless patients with tuberculosis]. Kekkaku:[Tuberculosis]. 2001;76(4):363-370.

39. Esterbauer H, Benedetti A, Lang J, Fulceri R, Fauler G, Comporti M. Studies on the mechanism of formation of 4-hydroxynonenal during microsomal lipid peroxidation. Biocbimica et Biophysica Acta (BBA)-Lipids and Lipid Metabolism. 1986;876(1):154-166.

40. Esterbauer H, Cheeseman K, Dianzani M, Poli G, Slater T. Separation and characterization of the aldehydic products of lipid peroxidation stimulated by ADP-Fe2+ in rat liver microsomes. Biochemical Journal. 1982;208(1):129-140.

41. Esterbauer H, Cheeseman KH. [42] Determination of aldehydic lipid peroxidation products: malonaldehyde and 4-hydroxynonenal. Methods in ensymology. Vol 186: Elsevier; 1990:407-421.

42. Esterbauer H, Schaur RJ, Zollner H. Chemistry and biochemistry of 4-hydroxynonenal, malonaldehyde and related aldehydes. Free Radical Biology and Medicine. 1991;11(1):81-128.

43. Sharma B. Oxidative stress in HIV patients receiving antiretroviral therapy. Current HIV Research. 2014;12(1):1321.

44. Hurwitz BE, Klimas NG, Llabre MM, et al. HIV, metabolic syndrome $\mathrm{X}$, inflammation, oxidative stress, and coronary heart disease risk. Cardiovascular Toxicology. 2004;4(3):303-315.

45. MandaKR, Banerjee A, Banks WA, Ercal N. Highly active antiretroviral therapy drug combination induces oxidative stress and mitochondrial dysfunction in immortalized human blood-brain barrier endothelial cells. Free Radical Biology and Medicine. 2011;50(7):801-810.

46. Mondal D, Pradhan L, Ali M, Agrawal KC. HAART drugs induce oxidative stress in human endothelial cells and increase endothelial recruitment of mononuclear cells. Cardiovascular Toxicology. 2004;4(3):287-302.

47. Nagiah S, Phulukdaree A, Chuturgoon A. Mitochondrial and oxidative stress response in HepG2 cells following acute and prolonged exposure to anti-retroviral drugs. Journal of Cellular Biochemistry. 2015;116(9):1939-1946.

48. Serpa J, Haque D, Valayam J, Breaux K, Rodriguez-Barradas MC. Effect of combination anti-retroviral treatment on total protein and calculated globulin levels among HIV-infected patients. International Journal of Infectious Diseases. 2010;14.

49. Wang X, Chai H, Lin PH, Yao Q, Chen C. Roles and mechanisms of human immunodeficiency virus protease inhibitor ritonavir and other anti-human immunodeficiency virus drugs in endothelial dysfunction of porcine pulmonary arteries and human pulmonary artery endothelial cells. The American Journal of Pathology. 2009;174(3):771-781.

50. Weiß M, Kost B, Renner-Müller I, Wolf E, Mylonas I, Brüning A. Efavirenz causes oxidative stress, endoplasmic reticulum stress, and autophagy in endothelial cells. Cardiovascular Toxicology. 2016;16(1):90-99.

51. Noori S. An overview of oxidative stress and antioxidant defensive system. Open Access Scientific Reports. 2012;1(8):1-9.

52. Tabe FN, Yanou NN, Kamdje AHN, Ntso A-SA. Oxidative Role of HIV/AIDS: Anti-retroviral Drugs and Medicinal Plants with Anti-HIV Activity. Journal of Diseases and Medicinal Plants. 2015;1(5):68-75.

53. Ngondi JL, Oben J, Forkah DM, Etame LH, Mbanya D. The effect of different combination therapies on oxidative stress markers in HIV infected patients in Cameroon. AIDS Research and Therapy. 2006;3(1):1.

54. Lee D-H, Gross MD, Jacobs DR. Association of se- 
rum carotenoids and tocopherols with $\gamma$-glutamyltransferase: the Cardiovascular Risk Development in Young Adults (CARDIA) study. Clinical chemistry. 2004;50(3):582588 .
55. Wamutu S, Francque S, Chatterjee S, et al. Vitamin $\mathrm{C}$ as well as $\beta$-carotene attenuates experimental liver fibrosis after intoxication with carbon tetrachloride in rats. International Journal of Biological and Chemical Sciences. 2012;6(2):559-571. 